\title{
Epoxide hydrolase conformational heterogeneity for the resolution of bulky pharmacologically-relevant epoxide substrates
}

\author{
Eila Serrano-Hervás, ${ }^{\dagger[a]}$ Guillem Casadevall, ${ }^{\dagger[a]}$ Marc Garcia-Borràs, ${ }^{[c]}$ Ferran Feixas, ${ }^{*[a]}$ and Sílvia \\ Osuna*[a,b]
}

\begin{abstract}
The conformational landscape of Bacillus megaterium epoxide hydrolase $(B m E H)$ and how it is altered by mutations that confer the enzyme the ability to accept bulky epoxide substrates has been investigated. Extensive molecular dynamics (MD) simulations coupled to active site volume calculations have unveiled relevant features of the enzyme conformational dynamics and function. Our long-timescale MD simulations identify a key conformational state not previously observed by means of X-ray crystallography and short MD simulations that presents the loop containing one of the catalytic residues Asp239 in a wide-open conformation, which is likely involved in the binding of the epoxide substrate. Introduction of mutations M145S and F128A dramatically alters the conformational landscape of the enzyme. These singly mutated variants are able to accept bulkier epoxide substrates due to the disorder in the $\alpha$-helix containing the catalytic Tyr144 and some parts of the lid domain induced by mutation. These changes impact the enzyme active site, which is substantially wider and more complementary to the bulky pharmacologically-relevant epoxide substrates.
\end{abstract}

Enzymes are inherently dynamic and sample alternative conformations to the native state. ${ }^{[1]}$ Many studies have shown that conformational heterogeneity is a key feature of enzymes, which is important for their function and for their evolution towards novel activities. ${ }^{[2]}$ The enzyme free energy landscape originates from the different conformations that exist in dynamic equilibrium, which if separated by small energy barriers can be significantly populated in solution. Mutations introduced in the enzyme, but also the binding of substrate, inhibitor or product can induce a redistribution of the populations of the conformational states that pre-exist in solution. ${ }^{[3]}$ The ability of some enzymes to accelerate additional promiscuous reactions has indeed been connected to this conformational plasticity and flexibility. ${ }^{[4]}$ Experimental evidences have been provided supporting the importance of enzyme conformational dynamics that include B-factor analysis, careful inspection of crystallographic structures along evolutionary pathways, changes in NMR spectra, among others. ${ }^{[2 a, 4 a, 5]}$ Computational methods have been shown to be particularly useful in this regard. ${ }^{[50,6]}$ We have recently found that molecular dynamics (MD) simulations coupled to correlation-based analysis can provide important insights on the alternative conformations that the enzyme can adopt in solution, but most importantly can predict which amino acid positions are key for favouring a desired population shift to enhance a promiscuous activity. ${ }^{[2]}$

Most of the epoxide hydrolases $(\mathrm{EH})$ structurally characterized up to date possess an $\alpha, \beta$-sheet core and a flexible lid domain that covers the enzyme active site (see Figure $1 \mathbf{A}$ ) ${ }^{[7]}$ In the lid, two catalytically relevant tyrosine residues are contained (Tyr144 and Tyr203), which are responsible for the recognition and activation of the epoxide substrate to yield the vicinal diol in high enantio- and regioselectivity (see Figure 1B). ${ }^{[7 c, 8]}$ In the core domain, EHs present a conserved catalytic triad (Asp97-His267-Asp239), as well as the HGXP oxyanion motif responsible for stabilizing the negative charge developed after the epoxide ring opening by the nucleophilic Asp 97. 


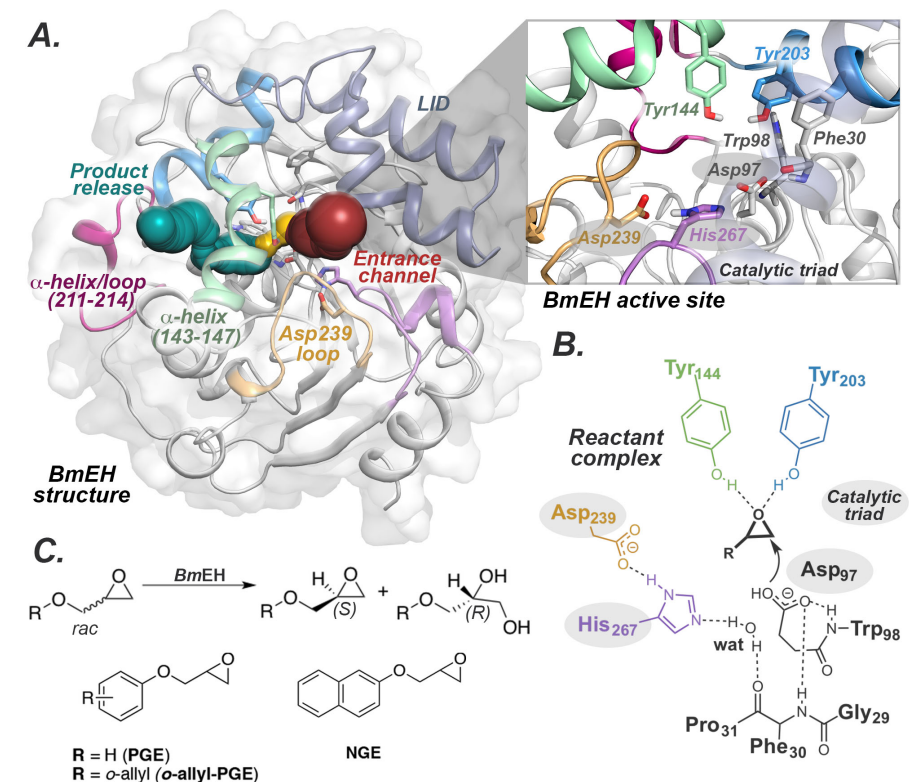

Figure 1. A) Representation of $B m E H$ enzyme wild-type structure (PDB: $4 \mathrm{NZZ}$ ), and active site access tunnels: substrate entrance (zone 1, in red), active site (zone 2, yellow) and product release (zone 3, teal). Relevant regions for the enzyme catalytic activity are highlighted: the loops containing the catalytic triad residues Asp239 (in gold), His267 (violet), and the nucleophilic Asp97 (grey); the $\alpha$-helices of Tyr144 and Tyr203 in green and blue, respectively. B) Schematic representation of BmEH active site with an epoxide substrate bound (reactant complex). C) General reaction scheme of epoxide hydrolysis to yield the corresponding vicinal diol, together with the representation of bulky pharmacologically-relevant epoxide substrates phenyl glycidyl ether (PGE) and naphthyl glycidyl ether (NGE) structures.

The mechanism followed by EHs for catalysing the hydration of racemic epoxides has been long debated. ${ }^{[9]}$ Many computational studies have been published in the literature providing a detailed atomistic description of the accepted two-step mechanism, and how active site mutations affect the activity and selectivity of the enzyme. ${ }^{[9]}$ In contrast to other reported $\mathrm{EHs},{ }^{\left[{ }^{[9 d}, 10\right]}$ Bacillus megaterium $\mathrm{EH}(\mathrm{BmEH})$ has an unusual (R)-selectivity, being also active towards ortho-substituted phenyl glycidyl ethers (PGE, see Figure 1C) providing excellent enantiomeric ratios. ${ }^{[11]}$ This makes $B m E H$ a potential industrial biocatalyst for the production of $\beta$-blocker drugs such as (S)-alprenolol and (S)-propranolol. ${ }^{[11 \mathrm{~b}]}$ In a recent study by our group, the origin of selectivity of $\mathrm{BmEH}$ towards racemic styrene oxide and its para-nitro derivative was computationally explored. ${ }^{[12]}$ Interestingly, substantially lower activation barriers were found for (S)-epoxides, indicating that the inherent $(R)$-selectivity of the enzyme can be modulated by the nature of the epoxide substrate as well.

$\mathrm{BmEH}$ was crystallized in the presence of phenoxyacetamide, a substrate analogue (PDB: 4O08). ${ }^{[1 \mathrm{~b}]}$ Interestingly, three different potential binding sites were identified corresponding to either the active site region, named zone 2 , that contains the abovementioned catalytic triad as well as the oxyanion motif, a deep cleft identified as zone 1 or entrance channel, and a bowl-like area corresponding to the product release or zone 3 (see active site tunnels highlighted in Figure 1A). Alanine scanning experiments at two positions located in the substrate entrance and product release zones, i.e. residues F128 and M145, were used to support the hypothesis of the different active site tunnels. ${ }^{[11 b]}$ Interestingly, mutation of both positions to smaller residues (serine and alanine) was found to be crucial for enhancing the catalytic activity of the enzyme towards the resolution of bulky epoxide substrates such as $\alpha$-naphthyl glycidyl ether (NGE, the activity is from 25 to 434 times higher than for the wild type enzyme). ${ }^{[11 a, b]}$ This increase in activity was 
attributed to the wider product release zone 3 of the variants that is supposed to facilitate the product dissociation step. ${ }^{[11 b]}$

The hypothesis that different access tunnels for the substrate association and product dissociation exist in $B m E H$, combined with its unique ability to accept bulkier substrates of industrial interest make the study of the conformational variability of $B m E H$ highly appealing. Of particular relevance is the evaluation of the role of positions F128 and M145. In this communication, we evaluate the conformational heterogeneity of $\mathrm{BmEH}$ by means of long-timescale molecular dynamics (MD) simulations, and explore how the included mutations affect the conformational landscape and the ability of the enzyme to accept bulkier pharmacologically-relevant epoxide substrates.

\section{BmEH conformational heterogeneity:}

The free energy landscape (FEL) constructed from an accumulated simulation time of 19 microseconds of MD simulations for the wild-type enzyme in the apo state is shown in Figure 2A. Dimensionality reduction of the highly complex MD dimensional data was achieved by applying the kinetically-relevant time-lagged independent components analysis (TICA) (see computational details and Scheme $\mathbf{S 1}$ for free energy construction). Wild-type EH can adopt four major conformations, among which the most populated one corresponds to the X-ray-like conformation $1^{\mathrm{WT}}$ (see Figure $2 \mathrm{~A}$ ). In this conformational state, the catalytic machinery of the enzyme is well-positioned for catalysis: both Tyr144 and Tyr203 are properly positioned, and the Asp293-His267-Asp97 triad is in a catalytically competent conformation for performing the nucleophilic attack after substrate binding (see Figure 1C). However, this conformational state presents quite small volumes in both region 1 and 3 (an approximate total volume of $133 \AA^{3}$ ). The other three conformational states sampled are not as catalytically competent as $1^{\text {WT }}$ as their catalytic triad arrangements present deviations from the ideal quantum mechanics geometry. ${ }^{[12]}$ However, in the absence of ligand they are also substantially populated in solution.

Conformational state $\mathbf{2}^{\mathrm{WT}}$ exhibits a different conformation of the sidechain of the oxyanion hole residue Phe30. This change of Phe30 affects the nearby residue Trp98, also involved in the stabilization of the developed negative charges during the course of the epoxide ring opening reaction. $\ln 3^{\mathrm{WT}}$, the lid that covers the active site of the enzyme is slightly deviated from the X-ray position. This displacement affects the loop that contains His267, which is still situated at a short distance from the catalytic Asp97 (at ca. 3.6 $\AA$ ). The loop containing Asp239 that completes the catalytic triad is also affected, and adopts a partially open conformation (see black arrows in Figure $2 \mathrm{~A}, 3^{\mathrm{WT}}$ ). Conformations $2^{\mathrm{WT}}$ and $3^{\mathrm{WT}}$ present substantially larger volumes as compared to the X-ray conformation $1^{\mathrm{WT}}$ (ca. 170 and $230 \AA^{3}$ for $2^{\mathrm{WT}}$ and $3^{\mathrm{WT}}$, respectively, see Table S1). Both conformational states are relatively stable and separated from $1^{\mathrm{WT}}$ by small energy barriers in this reaction coordinate. These results are indicating that in the absence of epoxide substrate, these three states are thermally accessible and coexist in solution. 
The observed displacement of the lid and Asp239-containing loop is even more pronounced in $4^{\mathrm{WT}}$ (see black arrows in Figure $2 \mathrm{~A}, 4^{\mathrm{WT}}$ ). Conformational state $4^{\mathrm{WT}}$ is quite stable and is separated from conformations $1^{\mathrm{WT}}-3^{\mathrm{WT}}$ by an energy barrier of ca. $3 \mathrm{kcal} / \mathrm{mol}$ in this reaction coordinate. The lid adopts a closed conformation substantially shifting the $\alpha$-helix that contains the catalytically relevant Tyr144, which is displaced from the active site. Tyr203 is also not properly positioned for catalysis as it locates its sidechain in region 3. The lid conformation has a dramatic impact into the loop containing Asp239 that adopts a catalytically unproductive open conformation. The calculation of the volume of zones 1 and 3 evidences that this conformational state $4^{\mathrm{WT}}$ has a wider access to the active site zone (a total volume of ca. $617 \AA^{3}$, see Table S1). These results are suggesting a key role of both the Asp239 loop and the coupled Tyr144 conformational change for binding the PGE epoxide substrate.

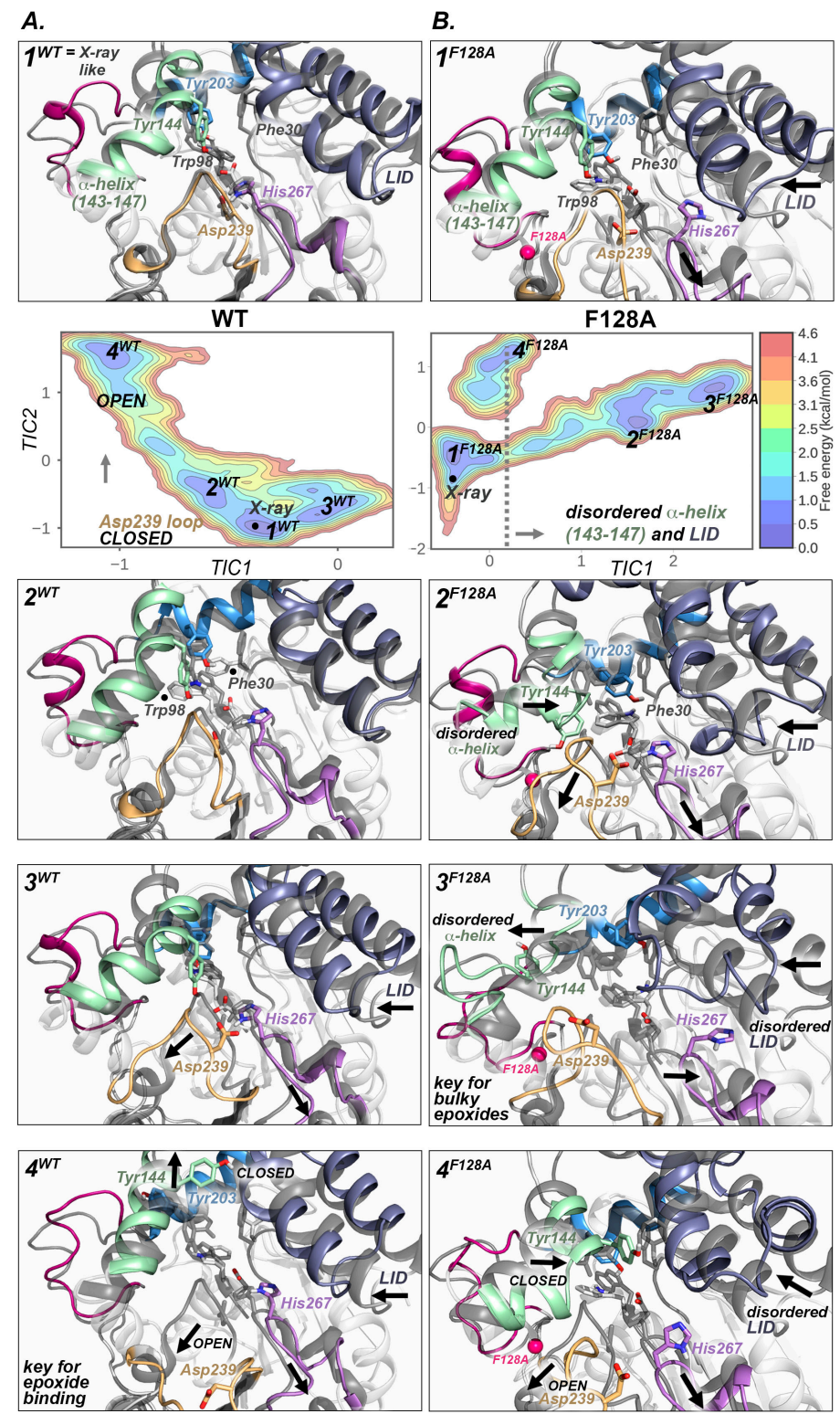

Figure 2. Representation of the free energy landscape obtained after applying the dimensionality reduction TICA to the accumulated MD data set for A) BmEH wild-type, and B) F128A variant. TIC1 describes the degree of $\alpha$-helix and lid disorder, while TIC2 differentiates open and closed conformational states of Asp239-containing loop. The four most stable conformational states for: A) BmEH wild-type (i.e. ${ }^{\text {wT }}$ - $4^{\text {WT }}$ ), and B) F128A variant (i.e. $1^{\mathrm{F} 128 \mathrm{~A}}-4^{\mathrm{F} 128 \mathrm{~A}}$ ) are overlaid to the crystallographic wild-type structure of BmEH (shown in gray). The most important changes occurring in each conformational state (as compared to the X-ray conformation) are highlighted with black arrows and bold text. 
Partial unfolding of Tyr144 alpha-helix is responsible for BmEH tolerance towards bulky epoxide substrates:

Mutations F128A and M145S were found to enhance the enzyme ability for accepting the bulkier o-allyl phenyl glycidyl ether (o-allyl-PGE) and naphthyl glycidyl ether (NGE) substrates, respectively. To elucidate the effect of the mutations in altering the conformational landscape of the wild-type enzyme, we ran additional MD simulations of both singly-mutated variants (see computational details).

The crystal structure available for the F128A variant (PDB: 4IO0) presents the diol product of NGE bound to zone 3 of the enzyme. As shown in Figure 2B, the X-ray conformation that presents all catalytic residues in a catalytically competent pose is not the most stable conformation of the enzyme in the apo state (see X-ray minima in Figure $2 \mathrm{~B}$ ). Instead, conformational state $1^{\mathrm{F} 128 \mathrm{~A}}$ is visited that presents the lid domain slightly shifted with respect to the crystal structure. This lid displacement affects the conformation of the loop containing His267 that positions its sidechain far away from the active site (the distance between $\mathrm{N} \varepsilon$ and Asp OD1 is ca. $7.7 \AA$ ). F128A mutation generates more space in zone 3, which is now occupied by the indole ring of Trp98 (see Figure 2B, $1^{\mathrm{F128A}}$ ). Apart from these deviations, the rest of the catalytic and oxyanion hole residues are properly positioned. This conformation has a similar volume to $1^{\mathrm{WT}}$.

Conformational state $2^{\mathrm{F128A}}$ is equally populated as $1^{\mathrm{F128A}}$, and is separated from $1^{\mathrm{F} 128 \mathrm{~A}}$ by an energy barrier of ca. $2.5 \mathrm{kcal} / \mathrm{mol}$ through different minor conformational states that present the $\alpha$-helix of Tyr144 slightly disordered (see black arrows in Figure $2 \mathrm{~B}, 2^{\mathrm{F} 128 \mathrm{~A}}$ ). Thus, converting $1^{\mathrm{F} 128 \mathrm{~A}}$ into $2^{\mathrm{F} 128 \mathrm{~A}}$ induces a partial disorder to the $\alpha$-helix of the catalytic Tyr144, which is displaced from the active site and occupies the space left by F128A mutation. $2^{\mathrm{F} 128 \mathrm{~A}}$ presents His267 closer to the nucleophilic Asp97 (at a distance between $\mathrm{N} \varepsilon$ and OD1 of ca. $5.8 \AA$ ), however as the loop that contains Asp239 is in a partially closed conformation the His267 *Asp267 distance is elongated up to ca. $7 \AA$. This conformation is separated from $3^{\mathrm{F} 128 \mathrm{~A}}$ by a small energy barrier of less than $1 \mathrm{kcal} / \mathrm{mol}$.

$3^{\mathrm{F128A}}$ presents the $\alpha$-helices of the lid and the helix that contains residues 143-147 highly disordered, as well as the Asp239 loop in a partially open conformation. These changes increase the volume of both zone 1 and 3 quite substantially leading to a total volume of ca. $631 \AA^{3}$ (see Table S1). As compared to the wild-type enzyme $4^{\mathrm{WT}}$, the wider volume found in region 1 suggest that conformational state $3^{\mathrm{F} 128 \mathrm{~A}}$ is likely to play a key role for binding the bulky NGE epoxide substrate and facilitate the diol product release. Conformational state $4^{\mathrm{F128A}}$ presents the lid in a closed conformation and the Asp239 loop open. Similarly to $4^{\mathrm{WT}}$, Tyr144 is displaced from the active site (see Figure 2). However, the computed volume for $4^{\mathrm{F} 128 \mathrm{~A}}$ is substantially smaller than in $\mathbf{4}^{\mathrm{WT}}$ (the total volumes are ca. 188 and $615 \AA^{3}$, respectively, see Table S1).

Our long-timescale MD simulations indicate that partial unfolding of the $\alpha$-helix that contains the catalytically relevant Tyr144 is required for $B m E H$ to accept the bulkier NGE epoxide substrate. Of special relevance is conformational state $3^{\mathrm{F128A}}$, not observed in the wild-type enzyme, that presents a wide active site pocket ready to bind NGE and facilitate the product release. The partial unfolding of Tyr144 $\alpha$-helix observed in our MD simulations is in line with the experimental observation that doubly mutated 
F128S/M145S variant presents severe protein-folding problems. ${ }^{[11 a]}$ To further evaluate our hypothesis, we decided to computationally evaluate the M145S variant.

The free energy landscape of variant M145S shares some common features of the wild-type enzyme and F128A variant (see Figure S2, SI). As observed in the wild-type enzyme, conformational state $1^{\text {M145S }}$ presents all catalytic residues in the proper conformation for catalysis, and $\mathbf{2}^{\mathrm{M} 145 \mathrm{~S}}$ has the oxyanion residues Phe30 and Trp98 with a different side-chain conformation. In this particular case, Trp98 can favorably position its indole ring in the space left by the M145S mutation. Conformational state $4^{\mathrm{M} 145 \mathrm{~S}}$ is also similar to $4^{\mathrm{WT}}$, as it presents the Asp239 loop in a wide-open conformation and Tyr144 displaced from the active site. This conformation presents a large volume of the active site (ca. $644 \AA^{3}$ ), which is bigger than for wild-type enzyme in line with the ability of M145S to accept o-allyl-PGE. An additional conformation is observed for M145S, i.e. $5^{\mathrm{M} 145 \mathrm{~S}}$, that resembles $4^{\mathrm{M} 145 \mathrm{~S}}$ although in this case a different conformation for the Trp98 sidechain is observed.

Interestingly M145S shares with F128A some conformational states that present the $\alpha$-helix of Tyr144 and the lid domain highly disordered. Although with the simulation time accumulated for M145S, the stable conformational states $2^{\mathrm{F} 128 \mathrm{~A}}$ and $3^{\mathrm{F} 128 \mathrm{~A}}$ observed in F128A could not be visited. Still, our findings for M145S are again demonstrating that both mutations induce partial unfolding of $\alpha$-helix 143-147, which generates a wider active site pocket and thus confers the enzyme the ability to accept bulky epoxide substrates.

\section{Conclusions}

Our extensive MD simulations combined with active site volume measurements reveal interesting features of $\mathrm{BmEH}$ conformational dynamics. A new conformational state is characterized for the first time that presents the loop containing the catalytic Asp239 in a wide-open conformation. This conformational change positions Tyr144 out of the active site, and thus leads to a wide active site and a broad substrate access channel. Based on these observations, we propose that this conformation is key for binding the epoxide substrate. Introduction of mutations M145S and F128A alters the conformational landscape of the enzyme. Of special relevance is the appearance of some major conformational states that present the $\alpha$ helix containing the catalytic Tyr144 highly disordered as well as some parts of the lid domain. These conformational states have a substantially wider active site pockets and access channels and thus correspond to the major reason for the ability of the M145S and F128A variants to accept bulkier pharmacologically-relevant epoxide substrates. Our study on the $B m E H$ conformational dynamics provides key relevant information for the pursue of new engineered variants with higher lid and $\alpha$-helix 143-147 plasticity for the resolution of bulky industrially-relevant epoxide substrates.

\section{Computational Section}

Molecular Dynamics Simulations. Long-timescale MD simulations in explicit water were performed using AMBER 16 package $^{[13]}$ in our in-house GPU cluster Galatea. The structures used were taken from the 
protein data bank (PDB); wild-type (WT, 4NZZ) and F128A variant complexed with the NGE-diol product (4IO0). M145S variant was generated by introducing the single mutation on different wild-type apo conformations explored through the long MD simulations using PyMOL (http://www.pymol.org). Each system was solvated in a pre-equilibrated truncated cuboid box with a 10- $\AA$ buffer of TIP3P water molecules and was neutralized by addition of explicit sodium counterions. All subsequent calculations were done using the widely tested Stony Brook modifications of the Amber 99 force field (ff99SB). The data used for the following analyses contained MD simulations of 2 trajectories of $5.2 \mu \mathrm{s}$ and 3 of $0.2 \mu \mathrm{s}$ for WT, 2 trajectories of 3.8 and $3.2 \mu \mathrm{s}$ and $0.2 \mu \mathrm{s}$ for $\mathrm{F} 128 \mathrm{~A}$ and 1 trajectory of $2 \mu \mathrm{s}$ for M145S starting from the X-ray conformation. Additional MD simulations were performed in multiple rounds starting from the FEL less populated clusters generated with $k$-means algorithm of the previous long MD simulations. The total time accumulated is $19 \mu$ s for WT, $20 \mu$ s F128A, and $18 \mu$ s for M145S (see Scheme S1 in SI).

FEL construction. The Free Energy Landscape (FEL) was constructed from the MD simulation data using pyEMMA ${ }^{[14]}$ (http://pyemma.org). We applied the dimensionality reduction technique time-lagged independent component analysis (TICA), ${ }^{[15]}$ by considering the minimum group distance between the $\mathrm{C} \alpha$ of every three residues as feature. TICA performs a dimensionality reduction by considering the time correlation of the data, thus minimizing the loss of kinetic information. The two slowest TICA components identified correspond to: TIC1 describing the disorder of $\alpha$-helix and LID domain, TIC2 accounting for Asp239-loop movement. TIC1 and TIC2 were used to construct the free energy landscape of the enzyme. Uniform distance clustering ( $\mathrm{k}$-means algorithm as implemented in pyEMMA ${ }^{[14]}$ ) was applied to obtain 30 clusters for WT and M145S, and 50 for F128A that clearly distinguished the different protein conformations explored along the MD simulations. The obtained clusters were further clusterized according to the $\mathrm{C} \alpha$ coordinates using cpptraj. ${ }^{[13]}$ Volume calculations were performed for the most populated and significant clusters with the computational tool POcket Volume MEasurer (POVME) 2.0. ${ }^{[16]}$ Full description of computational methods, and additional figures and tables as described in the text are provided in the Supporting Information (SI).

\section{Acknowledgements}

E. S.-H. thanks the Generalitat de Catalunya for a PhD fellowship (2017-FI-B-00118), M. G.-B is grateful to the Ramón Areces Foundation for a Postdoctoral Fellowship. F.F. thanks the European Community for MSCA-IF-2014-EF-661160-MetAccembly grant. S. O. thanks the Spanish MINECO CTQ2014-59212-P, Ramón y Cajal contract (RYC-2014-16846), the European Community for CIG project (PCIG14-GA-2013630978), and the funding from the European Research Council (ERC) under the European Union's Horizon 2020 research and innovation programme (ERC-2015-StG-679001). We thank the Generalitat de Catalunya for grup emergent 2017 SGR-1707. We are grateful for the computer resources, technical expertise, and assistance provided by the Barcelona Supercomputing Center - Centro Nacional de Supercomputación. 
[1] N. Tokuriki and D. S. Tawfik, Science 2009, 324, 203-207

[2] a) E. Campbell, M. Kaltenbach, G. J. Correy, P. D. Carr, B. T. Porebski, E. K. Livingstone, L. Afriat-Jurnou, A. M. Buckle, M. Weik, F. Hollfelder, N. Tokuriki and C. J. Jackson, Nat. Chem. Biol. 2016, 12, 944-950; b) B. Ma and R. Nussinov, Nat. Chem. Biol. 2016, 12, 890-891; c) A. Romero-Rivera, M. Garcia-Borràs and S. Osuna, ACS Catal. 2017, 7, 8524-8532.

[3] D. D. Boehr, R. Nussinov and P. E. Wright, Nat. Chem. Biol. 2009, 5, 789-796.

[4] a) E. C. Campbell, G. J. Correy, P. D. Mabbitt, A. M. Buckle, N. Tokuriki and C. J. Jackson, Curr. Opin. Struct. Biol. 2018, 50, 49-57; b) G. G. Hammes, S. J. Benkovic and S. Hammes-Schiffer, Biochem. 2011, 50, 10422-10430; c) A. Pabis, V. A. Risso, J. M. Sanchez-Ruiz and S. C. L. Kamerlin, Curr. Opin. Struct. Biol. 2018, 48, 83-92; d) A. Ramanathan, A. Savol, V. Burger, C. S. Chennubhotla and P. K. Agarwal, Acc. Chem. Res. 2014, 47, 149-156.

[5] a) J. M. Axe, E. M. Yezdimer, K. F. O’Rourke, N. E. Kerstetter, W. You, C. A. Chang and D. D. Boehr, J. Am. Chem. Soc. 2014, 136, 68186821 ; b) A. Neu, U. Neu, A.-L. Fuchs, B. Schlager and R. Sprangers, Nat. Chem. Biol. 2015, 11, 697-704; c) M. Orozco, Chem. Soc. Rev. 2014, 43, 5051-5066.

[6] a) A. Romero-Rivera, M. Garcia-Borràs and S. Osuna, Chem. Commun. 2017, 53, 284-297; b) J.-P. Colletier, A. Aleksandrov, N. Coquelle, S. Mraihi, E. Mendoza-Barberá, M. Field and D. Madern, Mol. Biol. Evol. 2012, 29, 1683-1694; c) G. Jiménez-Osés, S. Osuna, X. Gao, M. R. Sawaya, L. Gilson, S. J. Collier, G. W. Huisman, T. O. Yeates, Y. Tang and K. N. Houk, Nat. Chem. Biol. 2014, 10, 431-436; d) S. Osuna, G. Jiménez-Osés, E. L. Noey and K. N. Houk, Acc. Chem. Res. 2015, 48, 1080-1089.

[7] a) S. Barth, M. Fischer, R. D. Schmid and J. Pleiss, Proteins: Struct., Funct., Bioinf. 2004, 55, 846-855; b) D. Lindberg, A. Gogoll and M. Widersten, FEBS J. 2008, 275, 6309-6320; c) M. Nardini and B. W. Dijkstra, Curr. Opin. Struct. Biol. $1999,9,732-737$.

[8] a) R. N. Armstrong and C. S. Cassidy, Drug Metab. Rev. 2000, 32, 327-338; b) K. H. Hopmann and F. Himo, J. Phys. Chem. B. 2006, 110, 21299-21310.

[9] a) B. A. Amrein, P. Bauer, F. Duarte, A. J. Carlsson, A. Naworyta, S. L. Mowbray, M. Widersten and S. C. L. Kamerlin, ACS Catal. 2015, 5, 5702-5713; b) K. H. Hopmann, B. M. Hallberg and F. Himo, J. Am. Chem. Soc. 2005, 127, 14339-14347; c) K. H. Hopmann and F. Himo, Chem. Eur. J. 2006, 12, 6898-6909; d) M. T. Reetz, M. Bocola, L.-W. Wang, J. Sanchis, A. Cronin, M. Arand, J. Zou, A. Archelas, A.-L. Bottalla, A. Naworyta and S. L. Mowbray, J. Am. Chem. Soc. 2009, 131, 7334-7343; e) B. Schiott and T. C. Bruice, J. Am. Chem. Soc. 2002, 124, 14558-14570.

[10] a) Y. Xu, J.-H. Xu, J. Pan and Y.-F. Tang, Biotechnol Lett. 2004, 26, 1217-1221; b) M. T. Reetz, L. W. Wang and M. Bocola, Angew. Chem. 2006, 118, 1258-1263.

[11] a) X.-D. Kong, Q. Ma, J. Zhou, B.-B. Zeng and J.-H. Xu, Angew. Chem. Int. Ed. 2014, 53, 6641-6644; b) X.-D. Kong, S. Yuan, L. Li, S. Chen, J.-H. Xu and J. Zhou, Proc. Natl. Acad. Sci. U.S.A. 2014, 111, 15717-15722; c) J. Zhao, Y.-Y. Chu, A.-T. Li, X. Ju, X.-D. Kong, J. Pan, Y. Tang and J.-H. Xu, Adv. Synth. Catal. 2011, 353, 1510-1518.

[12] E. Serrano-Hervás, M. Garcia-Borràs and S. Osuna, Org. Biomol. Chem. 2017, 15, 8827-8835.

[13] D. A. Case, T. A. Darden, T. E. Cheatham, C. L. Simmerling, J. Wang, R. E. Duke, R. Luo, M. Crowley, R. C. Walker, W. Zhang, K. M. Merz, B. Wang, S. Hayik, A. Roitberg, G. Seabra, I. Kolossváry, K. F. Wong, F. Paesani, J. Vanicek, X. Wu, S. R. Brozell, T. Steinbrecher, H. Gohlke, L. Yang, C. Tan, J. Mongan, V. Hornak, G. Cui, D. H. Mathews, M. G. Seetin, C. Sagui, V. Babin and P. A. Kollman, AMBER 16, University of California, San Francisco, 2016.

[14] M. K. Scherer, B. Trendelkamp-Schroer, F. Paul, G. Pérez-Hernández, M. Hoffmann, N. Plattner, C. Wehmeyer, J.-H. Prinz and F. Noé, J. Chem. Theory Comput. 2015, 11, 5525-5542.

[15] a) G. Pérez-Hernández, F. Paul, T. Giorgino, G. D. Fabritiis and F. Noé, J. Chem. Phys. 2013, 139, 015102; b) C. R. Schwantes and V. S. Pande, J. Chem. Theory Comput. 2013, 9, 2000-2009.

[16] J. D. Durrant, L. Votapka, J. Sørensen and R. E. Amaro, J. Chem. Theory Comput. 2014, 10, 5047-5056. 